\title{
Metamaterial-Based Strain Sensors
}

\author{
Jining $\mathrm{Li}^{1,2}$, Withawat Withayachumnankul ${ }^{1,3}$, Shengjiang Chang ${ }^{2}$, and Derek Abbott ${ }^{1}$ \\ ${ }^{1}$ School of Electrical \& Electronic Engineering, The University of Adelaide, Adelaide, SA 5005, Australia \\ ${ }^{2}$ Institute of Modern Optics, Nankai University, Tianjin 300071, China \\ ${ }^{3}$ Faculty of Engineering, King Mongkut's Institute of Technology Ladkrabang, Bangkok 10240, Thailand \\ andrewli@eleceng.adelaide.edu.au
}

\begin{abstract}
In this paper, two metamaterial-based strain sensors at terahertz frequencies are introduced and simulated using CST Microwave Studio. They exhibit a large shift in the resonance frequency upon stretching or compression. They are very well suitable to monitoring and detecting mechanical deformation of a target structure remotely.
\end{abstract}

\section{INTRODUCTION}

Since artificial materials with unusual properties, such as negative refractive index, have been introduced to manipulate beams of light in different ways, the broad potential applications of metamaterials have emerged. The electromagnetic properties of these metamaterials are obtained from the underlying sub-wavelength periodic metallic structures resonating under the illumination of electromagnetic wave at a certain frequency [1]. Many different designs of subwavelength resonators have been investigated, for example: split ring resonators (SRRs) [2], electric LC resonators [3], pairs of rods [4], and fishnets [5]. Metamaterials provide a great deal of opportunities to realize superlenses, cloaking, perfect absorbers, antennas, and so on.

In all of these designs, the resonance of metamaterials is strongly dependent on the dimensions and geometry of the unit cell. A small modification to the structure dimension, particularly the parts where the field is concentrated, results in a large shift in the resonance frequency. Therefore, based on this dependent behavior between the deformation and frequency shift, the resonance frequency can be utilized to indicate the strain on an object with these subwavelength structures. This makes metamaterials very appropriate as strain sensors for non-intrusive telemetric sensing applications.

Since a long time ago, various types of strain sensors based on different principles have been developed, including fiberoptic sensors, piezoelectric sensors, carbon nanotube sensors and so on. In recent years, metamaterials have been utilized for the purpose of sensing. A strain sensor based on an SRR structure on a silicon substrate was demonstrated in the microwave range by R. Melik et al. in 2009 [6]. Another nested SRR strain sensor was designed by the same group in 2010 [7]. This structure combined multiple SRRs, or equivalently more capacitance, resulting in a sensitivity as high as $109 \mathrm{kHz} / \mathrm{kgf}$. The sensors were both fabricated as a gold pattern on a silicon substrate and fixed to the test materials with hard epoxy.

Lying between the microwave and infrared bands, terahertz radiation can penetrate dry, nonpolar and nonmetallic material, such as fabrics, woods, plastics, polymers and ceramics [8]. The radiation is hence considered very promising in nondestructive evaluation applications. Comparing to microwaves, terahertz waves yield a higher spatial resolution on a submillimetre level because of the shorter wavelength. Here we introduce strain sensors based on terahertz metamaterials. Inherent from the superior characteristics of terahertz radiation, the strain sensors in the terahertz regime is expected to operate with a high sensitivity, and high spatial resolution. Furthermore, the sensors can measure the strain in an object covered by terahertz-transparent materials.

In this paper, we present two designs for metamaterialinspired strain sensors, which are highly sensitive, structuresimplified, and easy to use. The first design is an I-shaped structure, suitable for single-axis strain sensing, and the other is a crossed-I structure for dual-axis sensing. The metal used to construct the metamaterials is gold, and the substrate is polydimethylsiloxane (PDMS). Compared to the rigid silicon substrate mentioned above, the PDMS substrate is low-cost, highly flexible and elastic, which can further improve the sensitivity of the sensors. In addition, owing to the surface tension of PDMS, the sensor can be attached to most of the smooth surfaces without additional adhesive. This self adhesion further benefits the sensitivity, as the deformation in the object can be directly transferred to the metallic resonators with only PDMS as an intermediate layer.

All the simulation in this article is performed using CST Microwave Studio. The electromagnetic properties of gold in terahertz range are calculated by using Drude model, with an epsilon infinity $\epsilon_{\infty}=1$, plasma frequency $\omega_{p}=1.37 \times 10^{16}$ $\mathrm{rad} / \mathrm{s}$, collision frequency $\gamma=6.45 \times 10^{12} \mathrm{~Hz}$ [9]. The PDMS substrate has a permittivity of approximately $\epsilon_{r}=2.55$ and conductivity of $2.5 \times 10^{-14} \mathrm{~S} / \mathrm{m}$ [10]. Periodic boundary conditions are utilized for the transverse boundaries to simulate an infinite planar array of resonators.

\section{DEVICE CONFIGURATION AND SIMULATION RESULTS}

\section{A. I-shaped strain sensor}

To simplify the structure and enhance the sensitivity, the I-shaped design was chosen. According to the previous investigation on a similar structure [11], the main absorption dip of this I-shaped structure in the transmission profile results from the electric resonance through strong capacitive coupling in the planar gap region between two unit cells. Therefore, the gap, 


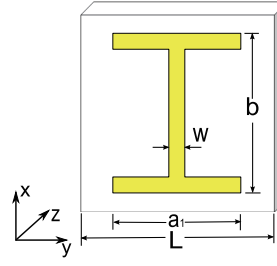

(a)

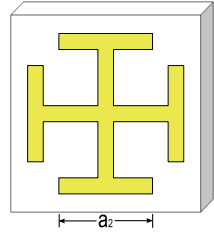

(b)

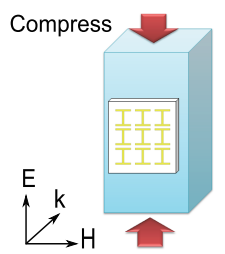

(c)
Fig. 1. (a) A unit cell of the I-shaped structure. (b) A unit cell of the crossingI structure. The physical parameters are as follow: $L=60 \mu \mathrm{m}, a_{1}=40 \mu \mathrm{m}$, $a_{2}=30 \mu \mathrm{m}, b=50 \mu \mathrm{m}, w=5 \mu \mathrm{m}$. The metal thickness is $200 \mathrm{~nm}$, and the substrate thickness is $100 \mu \mathrm{m}$. The terahertz wave propagates along the $z$ axis, with the polarization of the electric field along the $x$ axis. (c) Schematic showing an application of stress to the object.

defined as $g=L-b$, plays a crucial role in the resonance. This structure is suitable for measuring compression or elongation on one axis. A diagram of one unit cell with dimensions of the metallic resonator is shown in Fig. 1(a).

In the operation, the sensor is fixed onto the surface of the test object, and the force is applied along the $x$ axis, as shown in Fig. 1(c). When the terahertz wave is transmitted through the sensor, the incident electric field induces a large accumulation of surface charge in each arm of the I-structure. The charge leads to a strong electric field across the gap like a capacitor. The metallic resonator has a strong dipole resonance in the unit cells on the polarization of terahertz wave. The electric field distribution on resonance is shown in the inset of Fig. 2(a). The magnitude of the electric field established at the gap depends strongly on the arm length and is inversely proportional to the gap width. The arm length cannot change after fabrication, but a small change in the gap width will strongly affect the resonance frequency. The simulation results show that the resonance is at $1.17 \mathrm{THz}$, when the gap width is equal to $10 \mu \mathrm{m}$. After the pressure is applied to the object, the sensor deforms following the test object. Because the PDMS substrate is elastic except for the parts covered by metal, the deformation will be significant in the gap area. Due to a reduction in the gap width, the capacitance in the gaps is increasing. This change results in a red-shift in the resonance frequency. Fig. 2(a) shows the simulation results of the transmission with different gap widths from $10 \mu \mathrm{m}$ to $1 \mu \mathrm{m}$. The resonance frequency moves from $1.17 \mathrm{THz}$ to $0.86 \mathrm{THz}$. As shown in Fig. 2(b), the change of the resonance frequency increases from $11.6 \mathrm{GHz} / \mu \mathrm{m}$ to $304.5 \mathrm{GHz} / \mu \mathrm{m}$ for the change of gap width increasing from 1 $\mu \mathrm{m}$ to $9 \mu \mathrm{m}$. As the capacitance becomes larger, the frequency shift increases significantly. The maximum frequency shift per micron to the total shift is $32.38 \%$.

In the case of practical applications, it is possible that the object is not only under the compression along the $x$ axis, but also along the $y$ axis. In order to prove that the resonance frequency is sensitive to only a structural change from the deformation on the $x$ axis, the condition of compression on the $y$ axis is simulated. The results are shown in Fig. 3, in which $\Delta s$ is defined as the displacement distance on the $y$ axis from

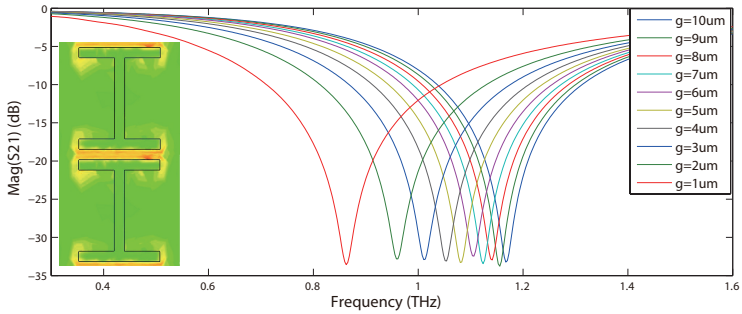

(a)

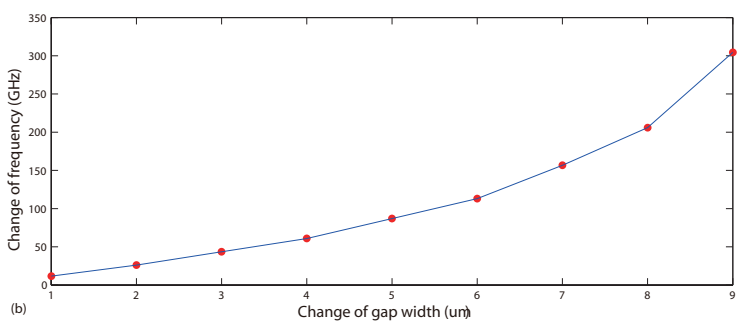

Fig. 2. (a) Transmission magnitude of I-shaped strain sensor for different gap widths from $10 \mu \mathrm{m}$ to $1 \mu \mathrm{m}$. The insert shows the the on-resonance maximum electric field distribution over two unit cells obtained from the simulation. (b) The change in the resonance frequency against the change of the gap width.

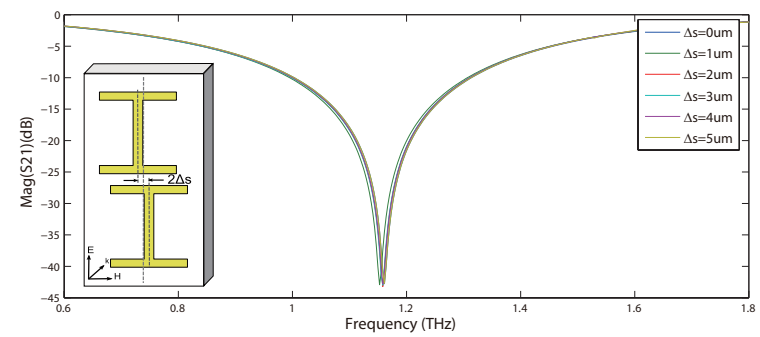

Fig. 3. Transmission magnitude of I-shaped strain sensor for different horizontal deformations from $0 \mu \mathrm{m}$ to $5 \mu \mathrm{m}$. The inset shows the definition of $\Delta s$ and the polarization of the incident terahertz wave.

the initial location from $0 \mu \mathrm{m}$ to $5 \mu \mathrm{m}$. The result demonstrates that the deformation on the $y$ axis has a negligible effect on the resonance frequency.

\section{B. Crossed-I strain sensor}

Based on the I-shaped sensor introduced above, we developed another sensor that is sensitive to the strain in the two orthogonal directions. The detailed structure and dimensions are shown in Fig. 1(b). To avoid connection between the arms, the length of each arm is decreased to $30 \mu \mathrm{m}$. This weakens the electric coupling in the gaps and leads to a slight blue shift in the resonance frequency [12]. The simulation shows the resonance at $1.34 \mathrm{THz}$. The process of measurement is similar to the I-shaped sensor. As shown in Fig. 4(a), the resonance frequency changes from $1.34 \mathrm{THz}$ to $1.01 \mathrm{THz}$ with different gap widths from $10 \mu \mathrm{m}$ to $1 \mu \mathrm{m}$. With the gap width decreasing, the capacitance in the gap enhances and the frequency shift increases, as shown in Fig. 4(b). The resonance frequency shift per micron to the total shift is $32.17 \%$. Because the terahertz wave is linearly polarized 

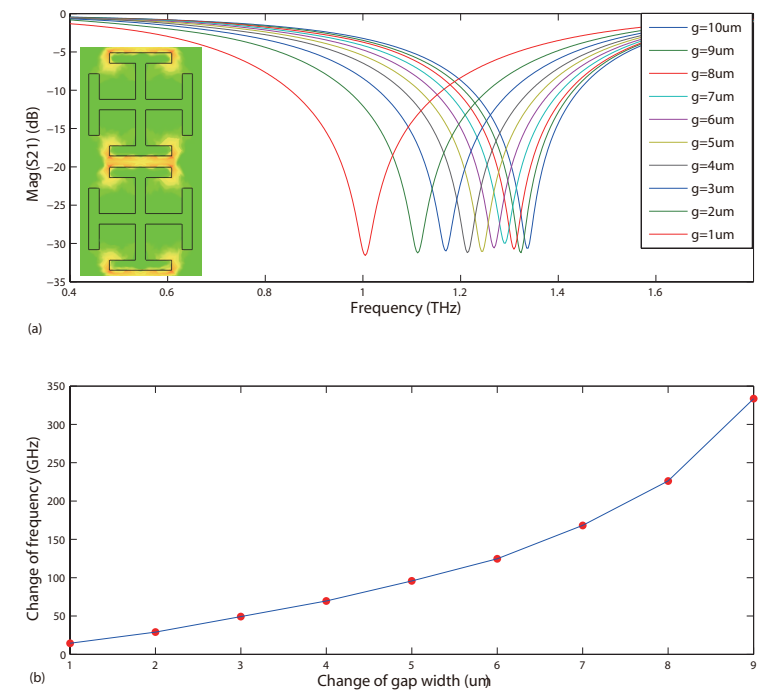

Fig. 4. (a) Transmission magnitude of the crossing I-shaped strain sensor for different gap widths from $10 \mu \mathrm{m}$ to $1 \mu \mathrm{m}$. The inset shows the on-resonance maximum electric field distribution over two unit cells obtained from the simulation. (b) The change of the resonance frequency against the change of the gap width.

and the resonance results from the gaps, the resonance is induced only in the gaps that are perpendicular to the incident electric-field polarization, as shown in the inset of Fig. 4(a). In other words, the resonance frequency only responds to the deformation along the polarization direction. Therefore, this sensor can be used to measure the strain independently on two directions along the $x$ and $y$ axes.

\section{CONCLUSION}

In summary, we present two designs of metamaterial-based strain sensors operating in the terahertz regime. Both of the sensors have a maximum sensitivity of $107 \mathrm{GHz}$ per micron under the applied stress. We will measure our sensors after fabrication using THz-TDS system. Future work will involve optimising the sensor design for a higher sensitivity.

\section{ACKNOWLEDGMENT}

The authors gratefully thank S. Sriram, M. Bhaskaran, and C. M. Shah of Microplatforms Research Group and Platform Technologies Research Institute, School of Electrical and Computer Engineering, RMIT University for useful discussions. This research was supported by the Australian Research Council Discovery Projects funding scheme under Project DP1095151 (W. Withayachumnankul) and DP1097281 (D. Abbott).

\section{REFERENCES}

[1] W. Withayachumnankul and D. Abbott, "Metamaterials in the terahertz regime," IEEE Photonics Journal, vol. 1, no. 2, pp. 99-118, 2009.

[2] J. B. Pendry, A. J. Robbins, and W. J. Stewart, "Magnetism from conductors and enhanced nonlinear phenomena," IEEE Transactions on Microwave Theory and Techniques, vol. 47, no. 11, pp. 2075-2084, 1999.

[3] D. Schurig, J. J. Mock, and D. R. Smith, "Electric-field-coupled resonators for negative permittivity metamaterials," Applied Physics Letters, vol. 88, no. 04, art. no. 041109, 2006.

[4] D. A. Powell, I. V. Shadrivov, and Y. S. Kivshar, "Cut-wire-pair structure as two-dimensional magnetic matamaterials," Optics Letters, vol. 16 , no. 19, pp. 15 185-15 190, 2008.

[5] S. Zhang, W. Fan, N. C. Panoiu, K. J. Malloy, R. M. Osgood, and S. R. J. Brueck, "Experimental demonstration of near-infrared negativeindex metamaterials," Physical Review Letters, vol. 95, art. no. 137404, 2005.

[6] R. Melik, E. Unal, N. K. Perkgoz, C. Puttlitz, and H. V. Demir, "Metamaterial-based wireless strain sensors," Applied Physics Letters, vol. 95, no. 01, art. no. 011106, 2009.

[7] R. Melik, E. Unal, N. K. Perkgoz, B. Santoni, D. Kamstock, C. Puttlitz, and H. V. Demir, "Nested metamaterials for wireless strain sensing," IEEE Journal of Selected Topics in Quantum Electronics, vol. 16, no. 2, pp. 450-458, 2010.

[8] D. Abbott and X. -C. Zhang, "Scanning the issue: T-ray imaging, sensing, and retection," Proceedings of the IEEE, vol. 95, no. 8, pp. 1509-1513, 2007.

[9] M. A. Ordal, R. J. Bell, R. W. Alexander, Jr, L. L. Long, and M. R. Querry, "Optical properties of fourteen metals in the infrared and far infrared: Al, $\mathrm{Co}, \mathrm{Cu}, \mathrm{Au}, \mathrm{Fe}, \mathrm{Pb}, \mathrm{Mo}, \mathrm{Ni}, \mathrm{Pd}, \mathrm{Pt}, \mathrm{Ag}, \mathrm{Ti}, \mathrm{V}$, and W," Applied Optics, vol. 24, no. 24, pp. 4493-4499, 1985.

[10] I. E. Khodasevych, C. M. Shah, W. S. T. Rowe, and A. Mitchell, "Flexible fishnet metamaterial on PDMS substrate for THz frequencies," in Optoelectronic and Microelectronic Materials and Devices (COMMAD), 2010, Dec. 2010, pp. 25-26.

[11] M. Choi, S. H. Lee, Y. Kim, S. B. Kang, J. Shin, M. H. Kwak, K. Y. Kang, Y. H. Lee, N. Park, and B. Min, "A terahertz metamaterials with unnaturally high refractive index," Nature, vol. 470, pp. 369-374, 2011.

[12] G. Donaelli, A. Vallecchi, F. Capolino, and A. Schuchinsky, "Metamaterial made of paired planar conductors: Particle resonances, phenomena and properties," Metamaterials, vol. 3, no. 1, pp. 10-27, 2009. 